\title{
Mathematical Appendix
}

\section{Ramsey Pricing}

\section{PROOF OF THEOREM 1:}

I maximize social welfare $V$ subject to $\pi>K$. The Lagrangian is

$$
V+\kappa(\pi-K)
$$

the associated first-order conditions are that for each $I$

$$
(1+\kappa)\left(P^{\mathcal{I}}-C^{\mathcal{I}}-c N^{\mathcal{J}}\right)-\kappa \mu^{\mathcal{I}}+\left(\overline{b^{\mathcal{J}}}+\kappa \widetilde{b^{\mathcal{J}}}\right) N^{\mathcal{J}}=0
$$

or

$$
P^{\mathcal{I}}-C^{\mathcal{I}}-c N^{\mathcal{J}}-\left(\frac{\kappa}{1+\kappa}\right) \mu^{\mathcal{I}}+\left(\left[\frac{1}{1+\kappa}\right] \overline{b^{\mathcal{J}}}+\left[\frac{\kappa}{1+\kappa}\right] \widetilde{b^{\mathcal{J}}}\right) N^{\mathcal{J}}=0
$$

or letting $\lambda \equiv \frac{\kappa}{1+\kappa}$

$$
P^{\mathcal{I}}=C^{\mathcal{I}}+c N^{\mathcal{J}}+\lambda \mu^{\mathcal{I}}-\left([1-\lambda] \overline{b^{\mathcal{J}}}+\lambda \widetilde{b^{\mathcal{J}}}\right) N^{\mathcal{J}}
$$

Then solve for $\lambda$ by substituting into the desired profit condition $\pi=K$ :

$$
\lambda\left(\mu^{\mathcal{A}} N^{\mathcal{A}}+\mu^{\mathcal{B}} N^{\mathcal{B}}+\left[\overline{b^{\mathcal{A}}}+\overline{b^{\mathcal{B}}}-\widetilde{b^{\mathcal{A}}}-\widetilde{b^{\mathcal{B}}}\right] N^{\mathcal{A}} N^{\mathcal{B}}\right)=K+\left(\overline{b^{\mathcal{A}}}+\overline{b^{\mathcal{B}}}-c\right) N^{\mathcal{A}} N^{\mathcal{B}}
$$

I now turn to the two alternative forms of the Ramsey problem mentioned in the text.

PROPOSITION 8: Interior Ramsey prices maximizing user surplus subject to the constraint that the firm makes a rate of return $r$ on her variable costs must solve

$$
P^{\mathcal{I}}=\underbrace{(1+r)\left(C^{\mathcal{I}}+c N^{\mathcal{J}}\right)}_{\text {cost-plus }}-\underbrace{N^{\mathcal{J}}\left(\lambda \widetilde{b^{\mathcal{J}}}+[1-\lambda] \overline{b^{\mathcal{J}}}\right)+\lambda \mu^{\mathcal{I}}}_{\text {two-sided classical Ramsey pricing (Theorem 1 }}
$$

where $\lambda \equiv \underbrace{\frac{\overbrace{\left(\overline{b^{\mathcal{A}}}+\overline{b^{\mathcal{B}}}-[1+r] c\right) N^{\mathcal{A}} N^{\mathcal{B}}}^{\text {subsidy (in cost-plus terms) }}}{N_{1}^{\mathcal{A}} \mu^{\mathcal{A}}+N^{\mathcal{B}} \mu^{\mathcal{B}}+\left(\overline{b^{\mathcal{A}}}+\overline{b^{\mathcal{A}}}-\widetilde{b^{\mathcal{A}}}-\widetilde{b^{\mathcal{B}}}\right) N^{\mathcal{A}} N^{\mathcal{B}}}}_{\text {demoninator from Theorem 1 }}$ 
PROOF:

The Lagrangian is

$$
V-(1-\kappa) \pi-r \kappa\left(c N^{\mathcal{A}} N^{\mathcal{B}}+C^{\mathcal{A}} N^{\mathcal{A}}+C^{\mathcal{B}} N^{\mathcal{B}}\right)
$$

with corresponding first-order conditions

$$
\kappa P^{\mathcal{I}}-\kappa(1+r)\left(C^{\mathcal{I}}+c N^{\mathcal{J}}\right)+(1-\kappa) \mu^{\mathcal{I}}+\left(\overline{b^{\mathcal{J}}}-[1-\kappa] \widetilde{b^{\mathcal{J}}}\right) N^{\mathcal{J}}=0
$$

or letting $\lambda \equiv\left(1-\frac{1}{\kappa}\right)$

$$
P^{\mathcal{I}}=(1+r)\left(C^{\mathcal{I}}+c N^{\mathcal{J}}\right)+\lambda \mu^{\mathcal{I}}-N^{\mathcal{J}}\left(\lambda \widetilde{b^{\mathcal{J}}}+[1-\lambda] \overline{b^{\mathcal{J}}}\right)
$$

Plugging into the rate-of-return constraint $\pi=r C$ yields

$$
\alpha\left(\mu^{\mathcal{A}} N^{\mathcal{A}}+\mu^{\mathcal{B}} N^{\mathcal{B}}+\left[\overline{b^{\mathcal{A}}}+\overline{b^{\mathcal{B}}}-\widetilde{b^{\mathcal{A}}}-\widetilde{b^{\mathcal{B}}}\right]\right)=\left(\overline{b^{\mathcal{A}}}+\overline{b^{\mathcal{B}}}-[1+r] c\right) N^{\mathcal{A}} N^{\mathcal{B}}
$$

which yields the desired solution.

Thus user Ramsey pricing with a required rate of return over variable costs is a natural combination of classical cost-plus regulation and the classical two-sided Ramsey pricing of Theorem 1. Prices mark-up costs by the required rate of return, incorporating two-sided internalization of cross-benefits and market power in the weighted-average fashion of classical Ramsey pricing above. Now that there is no required profit threshold, the weighting is given the amount necessary to offset the subsidy needed to achieve cost-plus Pigouvian pricing while maintaining the required rate of return. As noted earlier, if $r=0$ and the platform must simply break even, the solution is identical to the break-even classical OT pricing.

PROPOSITION 9: Interior Ramsey prices maximizing social surplus subject to the constraint that the firm makes a rate of return $r$ on her variable costs must solve

$$
P^{\mathcal{I}}=\underbrace{(1+\lambda r)\left(C^{\mathcal{I}}+c N^{\mathcal{J}}\right)}_{\text {cost-plus }}-\underbrace{N^{\mathcal{J}}\left(\lambda \overline{b^{\mathcal{J}}}+[1-\lambda] \widetilde{b^{\mathcal{J}}}\right)+\lambda \mu^{\mathcal{I}}}_{\text {two-sided classical Ramsey pricing (Theorem 1) }}
$$


where $\lambda \equiv \underbrace{\frac{\overbrace{r\left(C^{\mathcal{A}} N^{\mathcal{A}}+C^{\mathcal{B}} N^{\mathcal{B}}+c N^{\mathcal{A}} N^{\mathcal{B}}\right)}^{\text {target profits }}+\overbrace{\left(\overline{b^{\mathcal{A}}}+\overline{b^{\mathcal{B}}}-c\right) N^{\mathcal{A}} N^{\mathcal{B}}}^{\text {Pigouvian subsidy }}}{\left(r C^{\mathcal{A}}+\mu^{\mathcal{A}}\right) N^{\mathcal{A}}+\left(r C^{\mathcal{B}}+\mu^{\mathcal{B}}\right) N^{\mathcal{B}}+\left(\overline{b^{\mathcal{A}}}+\overline{b^{\mathcal{B}}}-\widetilde{b^{\mathcal{A}}}-\widetilde{b^{\mathcal{B}}}+2 r c\right) N^{\mathcal{A}} N^{\mathcal{B}}}}_{\text {(local) profit gain from moving towards rate-of-return maximization }}$

PROOF:

The Lagrangian is now

$$
V+\kappa\left(\pi-r\left[C^{\mathcal{A}} N^{\mathcal{A}}+C^{\mathcal{B}} N^{\mathcal{B}}+c N^{\mathcal{A}} N^{\mathcal{B}}\right]\right)
$$

giving first-order conditions

$$
(1+\kappa) P^{\mathcal{I}}-(1+\kappa[1+r]) C^{\mathcal{I}}-\kappa \mu^{\mathcal{I}}+\left(\overline{b^{\mathcal{I}}}+\kappa \widetilde{b^{\mathcal{I}}}\right) N^{\mathcal{J}}-(1+\kappa[1+r]) c N^{\mathcal{J}}
$$

Letting $\lambda \equiv \frac{\kappa}{1+\kappa}$ this takes the form in the text. Plugging this back into $\left(P^{\mathcal{A}}-[1+r] C^{\mathcal{A}}\right) N^{\mathcal{A}}+$ $\left(P^{\mathcal{B}}-[1+r] C^{\mathcal{B}}\right) N^{\mathcal{B}}-(1+r) c N^{\mathcal{A}} N^{\mathcal{B}}=0$ yields the definition for $\lambda$.

The social Ramsey pricing problem with a required rate of return over variable costs is a slight modification of user-optimal Ramsey pricing in Proposition 9.

\section{Generalization}

\section{PROOF OF THEOREM 2:}

I compute $V_{\mathcal{J}}^{\mathcal{I}}$. By definition

$$
\begin{aligned}
\left.V^{\mathcal{I}}(\mathbf{N})\right) & =\int_{\theta^{\mathcal{I}} \in \overline{\Theta^{\mathcal{I}}}}(\mathbf{N}) u^{\mathcal{I}}\left(\mathbf{N} ; \theta^{\mathcal{I}}\right) f^{\mathcal{I}}\left(\theta^{\mathcal{I}}\right) d \theta^{\mathcal{I}} \\
& =\int_{\theta^{\mathcal{I}}: u^{\mathcal{I}}\left(\mathbf{N} ; \theta^{\mathcal{I}}\right) \geq P^{\mathcal{I}}(\mathbf{N})} u^{\mathcal{I}}\left(\mathbf{N} ; \theta^{\mathcal{I}}\right) f^{\mathcal{I}}\left(\theta^{\mathcal{I}}\right) d \theta^{\mathcal{I}}
\end{aligned}
$$

Because $\widetilde{\Theta^{\mathcal{I}}}$ is the boundary of $\widetilde{\Theta^{\mathcal{I}}}$, by the Leibnitz Integral Rule

$V_{\mathcal{J}}^{\mathcal{I}}=\int_{\theta^{\mathcal{I}} \in \overline{\Theta^{\mathcal{I}}}} u_{\mathcal{J}}^{\mathcal{I}}\left(\mathbf{N} ; \theta^{\mathcal{I}}\right) f^{\mathcal{I}}\left(\theta^{\mathcal{I}}\right) d \theta^{\mathcal{I}}+$

$\int_{\theta^{\mathcal{I}} \in \widetilde{\Theta^{\mathcal{I}}}}\left(u_{\mathcal{J}}^{\mathcal{I}}\left[\mathbf{N} ; \theta^{\mathcal{I}}\right]-P_{\mathcal{J}}^{\mathcal{I}}[\mathbf{N}]\right) u^{\mathcal{I}}\left(\mathbf{N} ; \theta^{\mathcal{I}}\right) f^{\mathcal{I}}\left(\theta^{\mathcal{I}}\right) d \theta^{\mathcal{I}}=$

$$
\overline{u_{\mathcal{J}}^{\mathcal{I}}} N^{\mathcal{J}}+P^{\mathcal{I}} \int_{\theta^{\mathcal{I}} \in \widetilde{\Theta^{\mathcal{I}}}}\left(u_{\mathcal{J}}^{\mathcal{I}}\left[\mathbf{N} ; \theta^{\mathcal{I}}\right]-P_{\mathcal{J}}^{\mathcal{I}}[\mathbf{N}]\right) f^{\mathcal{I}}\left(\theta^{\mathcal{I}}\right) d \theta^{\mathcal{I}}
$$

By the implicit function theorem for $\mathcal{J} \neq \mathcal{I}$ and letting $\widetilde{N_{1}^{\mathcal{I}}} \equiv \frac{\partial \widetilde{N^{\mathcal{I}}}}{\partial P^{\mathcal{I}}}$

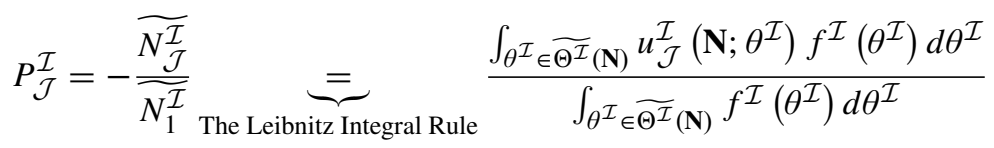


Thus term second term in A 3 vanishes for $\mathcal{I} \neq \mathcal{J}$. On other other hand for $\mathcal{I}=\mathcal{J}$ by the implicit function theorem

$$
P_{\mathcal{I}}^{\mathcal{I}}=\frac{\widetilde{N_{\mathcal{J}}^{\mathcal{I}}}-1}{\widetilde{N_{1}^{\mathcal{I}}}}=\frac{\int_{\theta^{\mathcal{I}} \in \widetilde{\Theta^{\mathcal{I}}}(\mathbf{N})} u_{\mathcal{J}}^{\mathcal{I}}\left(\mathbf{N} ; \theta^{\mathcal{I}}\right) f^{\mathcal{I}}\left(\theta^{\mathcal{I}}\right) d \theta^{\mathcal{I}}-1}{\int_{\theta^{\mathcal{I}} \in \widetilde{\Theta^{\mathcal{I}}}(\mathbf{N})} f^{\mathcal{I}}\left(\theta^{\mathcal{I}}\right) d \theta^{\mathcal{I}}}
$$

So the second term of A 3 is simply $P^{\mathcal{I}}$ (as the gross utility of all users in $\widetilde{\Theta^{\mathcal{I}}}$ ) is $P^{\mathcal{I}}$. Thus the first-order condition that marginal social benefit equal marginal cost becomes

$$
\begin{gathered}
\sum_{\mathcal{J}} V_{\mathcal{J}}^{\mathcal{I}}=C_{\mathcal{I}} \Longleftrightarrow \\
P^{\mathcal{I}}=C_{\mathcal{I}}-\sum_{\mathcal{J}} \overline{u_{\mathcal{I}}^{\mathcal{J}}} N^{\mathcal{J}}
\end{gathered}
$$

which is the Pigouvian condition reported in the text.

PROOF OF THEOREM 3:

Here I begin by computing marginal revenues. For $\mathcal{J} \neq I$ :

$$
R_{\mathcal{J}}^{\mathcal{I}}=\frac{\partial P^{\mathcal{I}}(\mathbf{N}) N^{\mathcal{I}}}{\partial N^{\mathcal{J}}}=P_{\mathcal{J}^{\mathcal{I}}} N^{\mathcal{I}}=\frac{\int_{\theta^{\mathcal{I}} \in \widetilde{\Theta^{\mathcal{I}}}(\mathbf{N})} u_{\mathcal{J}}^{\mathcal{I}}\left(\mathbf{N} ; \theta^{\mathcal{I}}\right) f^{\mathcal{I}}\left(\theta^{\mathcal{I}}\right) d \theta^{\mathcal{I}}}{\int_{\theta^{\mathcal{I}} \in \widetilde{\Theta^{\mathcal{I}}}(\mathbf{N})} f^{\mathcal{I}}\left(\theta^{\mathcal{I}}\right) d \theta^{\mathcal{I}}} N^{\mathcal{J}}=\widetilde{u_{\mathcal{J}}} N^{\mathcal{J}}
$$

while

$$
R_{\mathcal{I}}^{\mathcal{I}}=P^{\mathcal{I}}+P_{\mathcal{I}}^{\mathcal{I}} N^{\mathcal{I}}=P^{\mathcal{I}}+\widetilde{u_{\mathcal{I}}^{\mathcal{I}}} N^{\mathcal{I}}-\frac{P^{\mathcal{I}}}{\epsilon^{\mathcal{I}}}
$$

where $\epsilon^{\mathcal{I}} \equiv \frac{\partial \widetilde{N^{\mathcal{I}}}}{\partial P^{\mathcal{I}}} \frac{P^{\mathcal{I}}}{N^{\mathcal{I}}}$ is the price elasticity of participation (holding fixed anticipated participation). As usual, let $\mu^{\mathcal{I}} \equiv \frac{P^{\mathcal{I}}}{\epsilon^{\mathcal{I}}}$ be the market power, that is the difference between price and classical marginal revenue. Then equating marginal revenue to marginal cost requires

$$
P^{\mathcal{I}}-\mu^{\mathcal{I}}+\sum_{\mathcal{J}} \widetilde{u_{\mathcal{I}}^{\mathcal{J}}} N^{\mathcal{J}}=C_{\mathcal{I}}
$$

which can easily be re-arranged to yield either of the formulae in the theorem.

\section{Comparative Statics}

I begin by showing that $\rho^{\mathcal{I}}=-\frac{\mu^{\mathcal{I}}}{N^{\mathcal{I}} \frac{\partial^{2} \pi}{\partial \mathcal{I}^{2}}}$ and that local strict concavity of profits is equivalent to the local version of $2 \mathrm{SC}$, while violation of local weak 2SC implies local non-concavity. Then 
taking these facts as given, I establish Theorem 4.

$$
\left.\rho^{\mathcal{I}} \equiv \frac{d P^{\mathcal{I}}}{d C^{\mathcal{I}}}\right|_{N^{\mathcal{J}}}=\left.P_{1}^{\mathcal{I}} \frac{d N^{\mathcal{I}}}{d C^{\mathcal{I}}}\right|_{N^{\mathcal{J}}}=-\frac{\mu^{\mathcal{I}}}{N^{\mathcal{I}} \frac{\partial^{2} \pi}{\partial N^{\mathcal{I}^{2}}}}
$$

Thus $\rho^{\mathcal{I}}>(\geq) 0 \Longleftrightarrow \frac{\partial^{2} \pi^{2}}{\partial N^{\mathcal{I}^{2}}}<(\leq) 0$ and

$$
\frac{\partial^{2} \pi}{\partial N^{\mathcal{A}^{2}}} \frac{\partial^{2} \pi}{\partial N^{\mathcal{B}^{2}}}>(\geq) \frac{\partial^{2} \pi^{2}}{\partial N^{\mathcal{A}} \partial N^{\mathcal{B}}} \Longleftrightarrow \frac{\mu^{\mathcal{A}} \mu^{\mathcal{B}}}{N^{\mathcal{A}} N^{\mathcal{B}}}>(\geq) \rho^{\mathcal{A}} \rho^{\mathcal{B}} \chi^{2}
$$

which shows that strict local 2SC implies local concavity (by the classical Hessian test) and weak 2SC is necessary for local concavity. Based on these, Theorem 4 is easy to establish.

\section{Two-sided contraction}

\section{PROOF OF THEOREM 4:}

The forward direction of the proof follows directly from above: global concavity of profits (which is equivalent to the negative definiteness of profits, which is $2 \mathrm{SC}$ ) in $\left\langle N^{\mathcal{A}}, N^{\mathcal{B}}\right\rangle$ is independent of $\left\langle C^{\mathcal{A}}, C^{\mathcal{B}}\right\rangle$ by inspection. For the converse, suppose that 2SC were violated at some pair of participation rates $\left\langle\widetilde{N^{\mathcal{A}}}, \widetilde{N^{\mathcal{B}}}\right\rangle$. Let

$$
\widetilde{C^{\mathcal{I}}} \equiv P^{\mathcal{I}}-\mu^{\mathcal{I}}+\widetilde{b^{\mathcal{J}}} N^{\mathcal{J}}-c N^{\mathcal{J}}
$$

be evaluated at $\left\langle\widetilde{N^{\mathcal{A}}}, \widetilde{N^{\mathcal{B}}}\right\rangle$. Then clearly equation (2) given costs $\left\langle\widetilde{C^{\mathcal{A}}}, \widetilde{C^{\mathcal{B}}}\right\rangle$ has a solution at $\left\langle\widetilde{N^{\mathcal{A}}}, \widetilde{N^{\mathcal{B}}}\right\rangle$. However because $2 \mathrm{SC}$ is violated here, this must be a local minimum or saddle-point of the firm's profits. Therefore it cannot be the optimal participation pair.

In the Armstrong case, from equation (12) $\chi=b^{\mathcal{A}}+b^{\mathcal{B}}$ so 2SC becomes

$$
\frac{\mu^{\mathcal{A}} \mu^{\mathcal{B}}}{N^{\mathcal{A}} N^{\mathcal{B}}}>\rho^{\mathcal{A}} \rho^{\mathcal{B}}\left(b^{\mathcal{A}}+b^{\mathcal{B}}\right)^{2}
$$

In the special case of linear demand that is typically analyzed, $\frac{\mu^{\mathcal{I}}}{N^{\mathcal{I}}}=\frac{1}{\phi^{\mathcal{I}}}$ where $\phi^{\mathcal{I}}$ is the (constant) slope of demand. If we parameterize demand by $N^{\mathcal{I}}\left(P^{\mathcal{I}}, N^{\mathcal{J}}\right)=\frac{Q^{\mathcal{I}}\left(2 \widetilde{P^{\mathcal{I}}}-P^{\mathcal{I}}-b^{\mathcal{I}} N^{\mathcal{J}}\right)}{2 P^{\mathcal{I}}}$ so that $Q^{\mathcal{I}}$ demand at price 0 and $\widetilde{P^{\mathcal{I}}}$ is the optimal price when there is no two-sidedness and 
$C^{\mathcal{I}}=0$ then $\frac{1}{\sigma^{\mathcal{I}}}=\frac{Q^{\mathcal{I}}}{2 P^{\mathcal{I}}}$ so the condition simplifies, after a bit of algebra, to

$$
\frac{\left(b^{\mathcal{A}}+b^{\mathcal{B}}\right) Q^{\mathcal{A}} Q^{\mathcal{B}}}{\sqrt{\frac{Q^{\mathcal{A}} Q^{\mathcal{B}} \widetilde{P^{\mathcal{A}}}}{4}}}<8
$$

which is that the surplus generated by two-sidedness at zero price should not be more than 8 times the geometric mean of optimal profits on the two sides if there were no two-sidedness, as optimal profits for linear demand with this parameterization are $\frac{Q^{\mathcal{I}} \widetilde{P^{\mathcal{I}}}}{2}$.

\section{Pigouvian second-order conditions}

Now I derive the second-order conditions for the Pigouvian problem. The first derivative of social welfare $V$ with respect to participation on side $\mathcal{I}$ is

$$
P^{\mathcal{I}}-C^{\mathcal{I}}-c N^{\mathcal{J}}+\overline{b^{\mathcal{J}}} N^{\mathcal{J}}
$$

Taking the derivative with respect to $N^{\mathcal{I}}$ yields

$$
\begin{gathered}
P_{1}^{\mathcal{I}}+\bar{b}_{b^{\mathcal{J}}} N^{\mathcal{J}}=-\frac{\mu^{\mathcal{I}}}{N^{\mathcal{I}}}+\frac{\partial \int_{-\infty}^{\infty} \int_{P \mathcal{J}}^{\infty}\left(N^{\mathcal{J}}, N^{\mathcal{I}}\right)-b^{\mathcal{J}} N^{\mathcal{I}} b^{\mathcal{J}} f^{\mathcal{J}}\left(B^{\mathcal{J}}, b^{\mathcal{J}}\right) d B^{\mathcal{J}} d b^{\mathcal{J}}}{\partial N^{\mathcal{I}}}= \\
-\frac{\mu^{\mathcal{I}}}{N^{\mathcal{I}}}+\int_{-\infty}^{\infty}\left(b^{\mathcal{J}}-\widetilde{b^{\mathcal{J}}}\right) b^{\mathcal{J}} f^{\mathcal{I}}\left(P^{\mathcal{J}}\left[N^{\mathcal{J}}, N^{\mathcal{I}}\right]-b^{\mathcal{J}} N^{\mathcal{I}}, b^{\mathcal{J}}\right) d b^{\mathcal{J}} \\
=-\frac{\mu^{\mathcal{I}}}{N^{\mathcal{I}}}+\frac{N^{\mathcal{J}}}{\mu^{\mathcal{J}}}\left(\widetilde{b^{\mathcal{J}^{2}}}-\widetilde{b^{\mathcal{J}^{2}}}\right)=-\frac{\mu^{\mathcal{I}}}{N^{\mathcal{I}}}+\frac{N^{\mathcal{J}}}{\mu^{\mathcal{J}}}\left(\widetilde{b^{\mathcal{J}^{2}}}-\widetilde{b^{\mathcal{J}^{2}}}\right)
\end{gathered}
$$

Thus the own-second derivative condition for concavity is that on both sides $I$

$$
\frac{\mu^{\mathcal{A}} \mu^{\mathcal{B}}}{N^{\mathcal{A}} N^{\mathcal{B}}}>\widetilde{\sigma^{\mathcal{I}}}
$$

where $\widetilde{\sigma^{\mathcal{I}}}$ is the variance of interaction benefits among marginal users on side $\mathcal{I}$. The cross partial is then

$$
\begin{gathered}
P_{2}^{\mathcal{A}}-c+\frac{\partial \widetilde{b^{\mathcal{B}}} N^{\mathcal{B}}}{\partial N^{\mathcal{B}}}=\widetilde{b^{\mathcal{A}}}-c+\frac{\partial \int_{-\infty}^{\infty} \int_{P^{\mathcal{B}}\left(N^{\mathcal{B}}, N \mathcal{A}\right)-b^{\mathcal{B}} N^{\mathcal{A}}}^{\infty} b^{\mathcal{B}} f^{\mathcal{B}}\left(B^{\mathcal{B}}, b^{\mathcal{B}}\right) d B^{\mathcal{B}} d b^{\mathcal{B}}}{\partial N^{\mathcal{B}}}= \\
\widetilde{b^{\mathcal{A}}}-c+\frac{\mu^{\mathcal{B}} \int_{-\infty}^{\infty} b^{\mathcal{B}} f^{\mathcal{I}}\left(P^{\mathcal{B}}\left[N^{\mathcal{B}}, N^{\mathcal{A}}\right]-b^{\mathcal{B}} N^{\mathcal{A}}, b^{\mathcal{B}}\right) d b^{\mathcal{B}}}{N^{\mathcal{B}}}=\widetilde{b^{\mathcal{A}}}+\widetilde{b^{\mathcal{B}}}-c
\end{gathered}
$$


So the positive determinant condition requires

$$
\frac{\mu^{\mathcal{A}} \mu^{\mathcal{B}}}{N^{\mathcal{A}} N^{\mathcal{B}}}>\frac{\left(\widetilde{b^{\mathcal{A}}}+\widetilde{b^{\mathcal{B}}}-c\right)^{2}}{\left(\frac{N^{\mathcal{A}} N^{\mathcal{B}} \widetilde{\sigma^{\mathcal{A}}}}{\mu^{\mathcal{A}} \mu^{\mathcal{B}}}-1\right)\left(\frac{N^{\mathcal{A}} N^{\mathcal{B}} \widetilde{\sigma^{\mathcal{B}}}}{\mu^{\mathcal{A}} \mu^{\mathcal{B}}}-1\right)}
$$

If imposed globally, just as with $2 \mathrm{SC}$, these ensure sufficiency of first-order conditions. By the same argument as in Section III for 2SC, these conditions are weak in the sense of Theorem 4. In the RT2003 case, heterogeneity is unidimensional so all marginal users have the same preferences and $\widetilde{\sigma^{\mathcal{A}}}=\widetilde{\sigma^{\mathcal{B}}}=0$. Therefore inequality A 4 becomes

$$
m^{\mathcal{A}} m^{\mathcal{B}}>\left(p^{\mathcal{A}}+p^{\mathcal{B}}-c\right)^{2}
$$

as $p^{\mathcal{I}}=\widetilde{b^{\mathcal{I}}}$ as there are only interaction values. By the first-order condition for the Pigouvian problem in the RT2003 case $p^{\mathcal{A}}+p^{\mathcal{B}}-c=-\overline{s^{\mathcal{A}}}=-\overline{S^{\mathcal{B}}}$ so this becomes

$$
m^{\mathcal{A}} m^{\mathcal{B}}>\overline{s^{\mathcal{A}}} \cdot \overline{s^{\mathcal{B}}}=\overline{\rho^{\mathcal{A}}} \cdot \overline{\rho^{\mathcal{B}}} m^{\mathcal{A}} m^{\mathcal{A}} \Longleftrightarrow 1>\overline{\rho^{\mathcal{A}}} \cdot \overline{\rho^{\mathcal{B}}}
$$

which is my social second-order condition from E. Glen Weyl (2009). In the Armstrong case

$$
\frac{\mu^{\mathcal{A}} \mu^{\mathcal{B}}}{N^{\mathcal{A}} N^{\mathcal{B}}}>\left(b^{\mathcal{A}}+b^{\mathcal{B}}\right)^{2}
$$

In the linear case, when $\rho^{\mathcal{I}}=\frac{1}{2}$ this is exactly the private $2 \mathrm{SC}$, except four times stronger.

\section{Positive comparative statics}

PROOF OF THEOREM 5:

Letting $R^{\mathcal{I}}\left(N^{\mathcal{I}}, N^{\mathcal{J}}\right) \equiv P^{\mathcal{I}}\left(N^{\mathcal{I}}, N^{\mathcal{J}}\right) N^{\mathcal{I}}$ be revenue on side $\mathcal{I}$ the cross partial is

$$
\begin{aligned}
& \chi=R_{12}^{\mathcal{A}}+R_{12}^{\mathcal{B}}-c=\frac{\partial M R^{\mathcal{A}}}{\partial N^{\mathcal{B}}}+\frac{\partial M R^{\mathcal{B}}}{\partial N^{\mathcal{A}}}-c=\frac{\partial\left(P^{\mathcal{A}}+P_{1}^{\mathcal{A}} N^{\mathcal{A}}\right)}{\partial N^{\mathcal{B}}}+\frac{\partial\left(P^{\mathcal{B}}+P_{1}^{\mathcal{B}} N^{\mathcal{B}}\right)}{\partial N^{\mathcal{A}}}-c= \\
& P_{2}^{\mathcal{A}}+P_{12}^{\mathcal{A}} N^{\mathcal{A}}+P_{2}^{\mathcal{B}}+P_{12}^{\mathcal{B}} N^{\mathcal{B}}-c=\widetilde{b^{\mathcal{A}}}+\widetilde{b_{1}^{\mathcal{A}}} N^{\mathcal{A}}+\widetilde{b^{\mathcal{B}}}+\widetilde{b_{1}^{\mathcal{B}}} N^{\mathcal{B}}-c=\widetilde{b^{\mathcal{A}}}+\widetilde{b^{\mathcal{B}}}-c-\frac{\mu_{\widetilde{b}}^{\mathcal{A}}}{N^{\mathcal{B}}}-\frac{\mu_{\widetilde{b}}^{\mathcal{B}}}{N^{\mathcal{A}}}
\end{aligned}
$$

where $M R^{\mathcal{I}}$ is the classical marginal revenue on side $\mathcal{I}$. Thus there are substitutes (complements) 
if $\widetilde{b} N^{\mathcal{A}} N^{\mathcal{B}}>(<) \mu_{\widetilde{b}}$. But by equation (7), $\mu^{\mathcal{I}}=P^{\mathcal{I}}-C^{\mathcal{I}}+\left(\widetilde{b^{\mathcal{J}}}-c\right) N^{\mathcal{J}}$ thus

$$
\mu=2 \widetilde{b} N^{\mathcal{A}} N^{\mathcal{B}}+\left(\widetilde{B^{\mathcal{A}}}-C^{\mathcal{A}}\right) N^{\mathcal{A}}+\left(\widetilde{B^{\mathcal{B}}}-C^{\mathcal{B}}\right) N^{\mathcal{B}}=\pi+\widetilde{b} N^{\mathcal{A}} N^{\mathcal{B}}
$$

Clearly $\mu, \pi>0$ so dividing through there are substitutes (complements) if

$$
\begin{gathered}
\frac{\widetilde{b} N^{\mathcal{A}} N^{\mathcal{B}}}{\pi+\widetilde{b} N^{\mathcal{A}} N^{\mathcal{B}}}<(>) \frac{\mu_{\tilde{b}}}{\mu} \\
\Longleftrightarrow 1-\frac{\widetilde{b} N^{\mathcal{A}} N^{\mathcal{B}}}{\pi+\widetilde{b} N^{\mathcal{A}} N^{\mathcal{B}}}>(<) 1-\frac{\mu_{\widetilde{b}}}{\mu} \Longleftrightarrow \frac{\pi}{\pi+\widetilde{b} N^{\mathcal{A}} N^{\mathcal{B}}}>(<) \frac{\mu_{\widetilde{B}}}{\mu}
\end{gathered}
$$

If $\mu_{\widetilde{B}}<0$ then we clearly have substitutes. Otherwise this is equivalent to

$$
\frac{\pi+\widetilde{b} N^{\mathcal{A}} N^{\mathcal{B}}}{\pi}<(>) \frac{\mu_{\widetilde{b}}+\mu_{\widetilde{B}}}{\mu_{\widetilde{B}}} \Longleftrightarrow \frac{\widetilde{b} N^{\mathcal{A}} N^{\mathcal{B}}}{\pi}<(>) \frac{\mu_{\widetilde{b}}}{\mu_{\widetilde{B}}}
$$

\section{Normative comparative statics}

\section{PROOF OF THEOREM 6:}

By implicit differentiation of equation (1)

$$
\frac{d N^{\mathcal{I}}}{d C^{\mathcal{J}}}=-\frac{N^{\mathcal{I}} \rho^{\mathcal{I}} \chi \frac{d N^{\mathcal{J}}}{d C^{\mathcal{J}}}}{\mu^{\mathcal{I}}}
$$

So

$$
\begin{gathered}
\frac{d N^{\mathcal{I}}}{d C^{\mathcal{J}}}=-\frac{N^{\mathcal{I}} \rho^{\mathcal{I}} \chi}{\mu^{\mathcal{I}}} \\
\frac{d V^{\mathcal{I}}}{d C^{\mathcal{J}}}=V_{1}^{\mathcal{I}} \frac{d N^{\mathcal{I}}}{d C^{\mathcal{J}}}+V_{2}^{\mathcal{I}} \frac{d N^{\mathcal{J}}}{d C^{\mathcal{J}}} \propto-N^{\mathcal{I}}\left(\rho^{\mathcal{I}} \chi+\overline{b^{\mathcal{I}}}-\widetilde{b^{\mathcal{I}}}\right)
\end{gathered}
$$

where the constant of proportionality is positive by concavity of profits. Clearly the effect of an exogenous increase in side $\mathcal{J}$ participation, driven by cost, is the opposite of the effect of an increase in cost (in sign, which is all I am concerned with). 


\section{The Scale-Income Model}

In the SI model $b_{i}^{\mathcal{I}}=\beta^{\mathcal{I}} B_{i}^{\mathcal{I}}$. I therefore index a user's type by her membership benefit $B_{i}^{\mathcal{I}}$. A user $i$ on side $\mathcal{I}$ will participate if

$$
B_{i}^{\mathcal{I}}+\beta^{\mathcal{I}} B_{i}^{\mathcal{I}} N^{\mathcal{J}}>P^{\mathcal{I}}
$$

that is if $B_{i}^{\mathcal{I}}>\frac{P^{\mathcal{I}}}{1+\beta^{\mathcal{I}} N^{\mathcal{J}}}=\frac{v^{\mathcal{I}}\left(N^{\mathcal{J}}\right) P^{\mathcal{I}}}{\beta^{\mathcal{I}}}$ if $\frac{v^{\mathcal{I}}}{\beta^{\mathcal{I}}}$ is positive and if $B_{i}^{\mathcal{I}}<\frac{v^{\mathcal{I}}\left(N^{\mathcal{J}}\right) P^{\mathcal{I}}}{\beta^{\mathcal{I}}}$ if $\frac{v^{\mathcal{I}}}{\beta^{\mathcal{I}}}$ is negative. Intuitively, $v^{\mathcal{I}}$ (roughly) represents the fraction of heterogeneity that is along the interaction dimension; if $v^{\mathcal{I}}$ is negative, then as prices rise interaction values tend to fall. Dividing by $\beta^{\mathcal{I}}$ converts this into membership benefits. It may be either the large or small scale users that participate and they may have either positive or negative membership benefits. In what follows, I derive everything from first principles, as in unidimensional models my assumption of smoothness of $f^{\mathcal{I}}$ fails. All the results would follow identically from directly specializing my more general results, as they did in the RT2003 and Armstrong cases; however, for expositional purposes it is useful to show how direct analysis of a unidimensional model leads to the same results as specializing general results from the RT2006 model.

$$
N^{\mathcal{I}}\left(P^{\mathcal{I}}, N^{\mathcal{J}}\right)=1-F^{\mathcal{I}}\left(\frac{v^{\mathcal{I}}\left[N^{\mathcal{J}}\right] P^{\mathcal{I}}}{\beta^{\mathcal{I}}}\right) \text { if } \frac{v^{\mathcal{I}}}{\beta^{\mathcal{I}}}>0 \text { and } N^{\mathcal{I}}=F^{\mathcal{I}} \text { if } \frac{v^{\mathcal{I}}}{\beta^{\mathcal{I}}}<0 \text { where } F^{\mathcal{I}} \text { is }
$$

the c.d.f. of membership values. Let $D^{\mathcal{I}}\left(B^{\mathcal{I}}\right) \equiv 1-F^{\mathcal{I}}$ or $F^{\mathcal{I}}$ as appropriate. Then $N_{1}^{\mathcal{I}}=$ $-\left|\frac{v^{\mathcal{I}}}{\beta^{\mathcal{I}}}\right| f^{\mathcal{I}}$, where $f^{\mathcal{I}}=F^{\mathcal{I}^{\prime}}$, and $N_{2}^{\mathcal{I}}=-\left(\frac{v^{\mathcal{I}}}{\beta^{\mathcal{I}}}\right) \frac{v^{\mathcal{I}^{\prime}} P^{\mathcal{I}}}{\beta^{\mathcal{I}}} f^{\mathcal{I}}$. By the definition, $v^{\mathcal{I}^{\prime}}=-\left(v^{\mathcal{I}}\right)^{2}$, yielding

$$
N_{2}^{\mathcal{I}}=\left(\frac{v^{\mathcal{I}}}{\beta^{\mathcal{I}}}\right) \frac{P^{\mathcal{I}}\left(v^{\mathcal{I}}\right)^{2}}{\beta^{\mathcal{I}}}=v^{\mathcal{I}} P^{\mathcal{I}}\left|\frac{v^{\mathcal{I}}}{\beta^{\mathcal{I}}}\right|
$$

Letting $P^{\mathcal{I}}\left(N^{\mathcal{I}}, N^{\mathcal{J}}\right)$ be the inverse of $N^{\mathcal{I}}$ with respect to its first argument, by the inverse and implicit function theorems we have $P_{1}^{\mathcal{I}}=-\frac{\mu^{\mathcal{I}}}{N^{\mathcal{I}}}$ where $\mu^{\mathcal{I}} \equiv \frac{N^{\mathcal{I}}}{f^{\mathcal{I}}}\left|\frac{\beta^{\mathcal{I}}}{v^{\mathcal{I}}}\right|$ and $P_{2}^{\mathcal{I}}=v^{\mathcal{I}} P^{\mathcal{I}}=\widetilde{b^{\mathcal{I}}}=$ $\beta^{\mathcal{I}} D^{\mathcal{I}^{-1}}\left(N^{\mathcal{I}}\right)=\beta^{\mathcal{I}} \widetilde{b^{\mathcal{I}}}$. Surplus on side $\mathcal{I}$ is

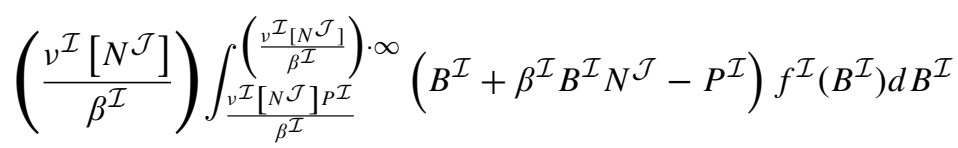

Using the change of variables $\phi^{\mathcal{I}}=\frac{B^{\mathcal{I}} \beta^{\mathcal{I}}}{v^{\mathcal{I}}}$ this becomes

$$
V^{\mathcal{I}}=\left|\frac{v^{\mathcal{I}}}{\beta^{\mathcal{I}}}\right| \int_{P^{\mathcal{I}}}^{\infty}\left(\phi^{\mathcal{I}}-P^{\mathcal{I}}\right) f^{\mathcal{I}}\left(\frac{v^{\mathcal{I}} \phi^{\mathcal{I}}}{\beta^{\mathcal{I}}}\right) d \phi^{\mathcal{I}}=\int_{P^{\mathcal{I}}}^{\infty} D^{\mathcal{I}}\left(\frac{v^{\mathcal{I}} \phi^{\mathcal{I}}}{\beta^{\mathcal{I}}}\right) d \phi^{\mathcal{I}}
$$


by the standard integration by parts for surplus. Therefore $V_{1}^{\mathcal{I}}=\mu^{\mathcal{I}}$ and

$$
\begin{gathered}
V_{2}^{\mathcal{I}}=-\int_{P^{\mathcal{I}}}^{\infty} \frac{v^{\mathcal{I}^{\prime}} \phi^{\mathcal{I}}}{\beta^{\mathcal{I}}}\left(\frac{v^{\mathcal{I}}}{\beta^{\mathcal{I}}}\right) f^{\mathcal{I}} d \phi^{\mathcal{I}}-v^{\mathcal{I}} P^{\mathcal{I}} N^{\mathcal{I}}= \\
\int_{P^{\mathcal{I}}}^{\infty} v^{\mathcal{I}} \phi^{\mathcal{I}}\left|\frac{v^{\mathcal{I}}}{\beta^{\mathcal{I}}}\right| f^{\mathcal{I}} d \phi^{\mathcal{I}}-v^{\mathcal{I}} P^{\mathcal{I}} N^{\mathcal{I}}=v^{\mathcal{I}} V^{\mathcal{I}}
\end{gathered}
$$

By E. Glen Weyl and Michal Fabinger's (2009)'s Theorem 3, $V^{\mathcal{I}}=N^{\mathcal{I}} \widehat{\mu^{\mathcal{I}}} \widehat{\rho^{\mathcal{I}}}$ where $\widehat{\mu^{\mathcal{I}}}$ is the inverse hazard rate of $D^{\mathcal{I}}$ with respect to $\phi$ at $\phi=P^{\mathcal{I}}$ and $\widehat{\rho^{\mathcal{I}}}$ is an average of $\frac{1}{1-\frac{\partial \mathcal{I I}^{\mathcal{I}}}{\partial N^{\mathcal{I}}} \frac{1}{P_{1}^{\mathcal{I}}}}$ over $\phi>P^{\mathcal{I}}$. Therefore to establish the formulae given in the text, I must show that, in fact, $\widehat{\mu^{\mathcal{I}}}=\mu^{\mathcal{I}}$ and $\widehat{\rho^{\mathcal{I}}}=\overline{\rho^{\mathcal{I}}}$.

$$
\widehat{\mu^{\mathcal{I}}}=-\frac{N^{\mathcal{I}}}{\left.\frac{\partial D^{\mathcal{I}}}{\partial \phi^{\mathcal{I}}}\right|_{\phi^{\mathcal{I}}=P^{\mathcal{I}}}}=\frac{N^{\mathcal{I}}}{f^{\mathcal{I}}}\left|\frac{\beta^{\mathcal{I}}}{v^{\mathcal{I}}}\right|=\mu^{\mathcal{I}}
$$

For the equivalence of $\widehat{\rho^{\mathcal{I}}}$ to an average of $\rho^{\mathcal{I}}$, let me calculate $\rho^{\mathcal{I}}$.

$$
\rho^{\mathcal{I}}=-\frac{\mu^{\mathcal{I}}}{N^{\mathcal{I}} \frac{\partial^{2} \pi}{\partial N^{\mathcal{I}^{2}}}}=-\frac{\mu^{\mathcal{I}}}{N^{\mathcal{I}}\left(P_{1}^{\mathcal{I}}-\mu_{1}^{\mathcal{I}}\right)}=\widehat{\rho^{\mathcal{I}}}
$$

Following a similar logic

$$
\begin{gathered}
\chi=P_{2}^{\mathcal{A}}+v^{\mathcal{B}} N^{\mathcal{B}} P_{1}^{\mathcal{B}}+v^{\mathcal{B}} P^{\mathcal{B}}-\mu_{2}^{\mathcal{I}}-c=v^{\mathcal{A}} P^{\mathcal{A}}+v^{\mathcal{B}} P^{\mathcal{B}}-c-v^{\mathcal{B}} N^{\mathcal{B}} \frac{\mu^{\mathcal{B}}}{N^{\mathcal{B}}}-\mu_{2}^{\mathcal{I}} \\
\mu_{2}^{\mathcal{I}}=\frac{\partial \frac{N^{\mathcal{I}}\left|\beta^{\mathcal{I}}\right|}{f^{\mathcal{I}}\left(N^{\mathcal{I}}\right)} \frac{1}{\partial N^{\mathcal{J}}}\left[\nu^{\mathcal{I}}\right] \mid}{N^{\mathcal{I}}}=\frac{N^{\mathcal{I}}\left|\beta^{\mathcal{I}}\right| \partial\left|\frac{1}{\beta^{\mathcal{I}}}+N^{\mathcal{J}}\right|}{\partial N^{\mathcal{I}}}=\left(v^{\mathcal{I}}\right) \frac{N^{\mathcal{I}}\left|\beta^{\mathcal{I}}\right|}{f^{\mathcal{I}}}=v^{\mathcal{I}} \mu^{\mathcal{I}}
\end{gathered}
$$

therefore

$$
\chi=\sum_{\mathcal{I}=A, B}\left(v^{\mathcal{I}}\left[P^{\mathcal{I}}-\mu^{\mathcal{I}}\right]\right)-c
$$

Because the natural analogs of the naïve simplifications from above are now apparent, I revert to directly using these, but direct derivations are available on request. 
The sign of equilibrium network effects from side $\mathcal{I}$ to side $\mathcal{J}$ are now determined by

$$
\nu^{\mathcal{J}} \mu^{\mathcal{J}} \overline{\rho^{\mathcal{J}}}+\rho^{\mathcal{J}}\left(\sum_{K=A, B}\left[v^{K}\left(P^{K}-\mu^{K}\right)\right]-c\right)
$$

As stated in the text, if the second term is positive (as in the newspaper case) and the first term also is (as on the advertiser side, because $\nu^{\mathcal{B}}>0$ ) then clearly equilibrium externalities from readers to advertisers are positive. On the reader's side the second term is positive, but the first is negative as $v^{\mathcal{A}}<0$. The equilibrium network effect will tend to be positive when complementarity is large relative to reader-side market power and when pass-through is decreasing, so that inframarginal harms are small relative to the pass-through of marginal cost changes.

The own-side cost-welfare effect on side $\mathcal{I}$

$$
\begin{gathered}
-\left(\mu^{\mathcal{A}} \mu^{\mathcal{B}}+\left[\sum_{K=A, B}\left(v^{K}\left[P^{K}-\mu^{K}\right]\right)-c\right] \rho^{\mathcal{J}} v^{\mathcal{I}} \mu^{\mathcal{I}} \rho^{\mathcal{I}} N^{\mathcal{A}} N^{\mathcal{B}}\right) \propto \\
-\left(\mu^{\mathcal{J}}+v^{\mathcal{I}} \rho^{\mathcal{J}} \overline{\rho^{\mathcal{I}}} N^{\mathcal{A}} N^{\mathcal{B}}\left[\sum_{K=A, B}\left(v^{K}\left[P^{K}-\mu^{K}\right]\right)-c\right]\right)
\end{gathered}
$$

For advertisers this is clearly negative as both terms are. For readers this is again ambiguous: while they lose from the higher prices the gain from resulting reduction in the Spence distortion and therefore advertising. Thus it is not clear that readers will be worse off as the newspaper business becomes less competitive, because on both sides of the market reduced competition is likely to lead to higher advertising rates and this may improve reader welfare.

\section{Applications}

In this appendix I describe formally the GASH model of duopoly, derive equilibrium conditions for it and use these to state formally and prove the results I referred to in Subsection VI.C. Armstrong only considers a linear demand, product differentiated model where all users must choose one platform in the spirit of Harold Hotelling (1929), making it impossible to compare monopoly to competition as under monopoly the platform would charge infinite prices.

\section{Demand}

Users on each side $\mathcal{I}$ have a usage benefit $b^{\mathcal{I}}$ which depends only on which side of the market they are on, not which platform they use nor their identity. users have a membership benefit for 
each platform 1 and $2, B_{i}^{\mathcal{I}, 1}$ and $B_{i}^{\mathcal{I}, 2}$ respectively, drawn from a symmetric density function $g^{\mathcal{I}}: R^{2} \rightarrow R$. Given a vector, consisting of a pair of side $\mathcal{I}$ prices and a pair of participation levels on side $\mathcal{J},\left(P^{\mathcal{I}, i}, P^{\mathcal{I},-i}, N^{\mathcal{J}, i}, N^{\mathcal{J},-i}\right)$, we can write the number of side $\mathcal{I}$ agents that choose to participate on platform $i$. Let us call this $N^{\mathcal{I}, i}$, where

$$
\begin{aligned}
N^{\mathcal{I}, i}\left(P^{\mathcal{I}, i}, P^{\mathcal{I},-i}, N^{\mathcal{J}, i}, N^{\mathcal{J},-i}\right)= \\
\quad \int_{P^{\mathcal{I}, i}-b^{\mathcal{I}} N^{\mathcal{J}, i}}^{\infty} \int_{-\infty}^{\mathcal{B}^{\mathcal{I}, i}+b^{\mathcal{I}} N^{\mathcal{J}, i}-P^{\mathcal{I}, i}+P^{\mathcal{I},-i}-b^{\mathcal{I}} N^{\mathcal{J},-i}} g^{\mathcal{I}}\left(B^{\mathcal{I}, i}, B^{\mathcal{I},-i}\right) d B^{\mathcal{I},-i} d B^{\mathcal{I}, i} .
\end{aligned}
$$

Let $R^{\mathcal{I}, i} \equiv b^{\mathcal{I}} N^{\mathcal{J}, i}-P^{\mathcal{I}, i}$ be the payoff an agent on side $\mathcal{I}$ receives from joining platform $i$, net of membership benefits. We can then express the number of side $\mathcal{I}$ agents that participate of platform $i$ as $N^{\mathcal{I}, i}=N^{\mathcal{I}, i}\left(P^{\mathcal{I}, i}, N^{\mathcal{J}, i}, R^{\mathcal{I},-i}\right)$, where

$$
\begin{aligned}
& N^{\mathcal{I}, i}\left(P^{\mathcal{I}, i}, N^{\mathcal{J}, i}, R^{\mathcal{I},-i}\right)= \\
& \int_{P^{\mathcal{I}, i}-b^{\mathcal{I}} N^{\mathcal{J}, i}}^{\infty} \int_{-\infty}^{\mathcal{B}^{\mathcal{I}, i}+b^{\mathcal{I}} N^{\mathcal{J}, i}-P^{\mathcal{I}, i}-R^{\mathcal{I},-i}} g^{\mathcal{I}}\left(B^{\mathcal{I}, i}, B^{\mathcal{I},-i}\right) d B^{\mathcal{I},-i} d B^{\mathcal{I}, i} .
\end{aligned}
$$

Note that as $N^{\mathcal{I}, i}$ is strictly decreasing in it's first argument, it can be inverted, yielding a well defined inverse demand $P^{\mathcal{I}, i}\left(N^{\mathcal{I}, i}, N^{\mathcal{J}, i}, R^{\mathcal{I},-i}\right)$.

\section{Duopoly equilibrium}

Supposing that both firm 1 and firm 2 offer insulating tariffs on both sides of the market; that is, $P^{\mathcal{I}, i}\left(P^{\mathcal{J}, i}\right)=b^{\mathcal{I}} N^{\mathcal{J}, i}+h^{\mathcal{I}, i}$ for some chosen $h^{\mathcal{I}, i}$ as the insulating tariff for the Armstrong model is just full insurance. We can the consider each firm $i$ to choose participation levels on each side, taking as given $R^{\mathcal{A},-i}$ and $R^{\mathcal{B},-i}$. Thus, in equilibrium, each firm maximizes, with respect to $N^{\mathcal{I}, i}, I=A, B$,

$$
\left[P^{\mathcal{A}, i}\left(N^{\mathcal{A}, i}, N^{\mathcal{B}, i}, R^{\mathcal{A},-i}\right)-C^{\mathcal{A}}\right] N^{\mathcal{A}, i}+\left[P^{\mathcal{B}, i}\left(N^{\mathcal{B}, i}, N^{\mathcal{A}, i}, R^{\mathcal{B},-i}\right)-C^{\mathcal{B}}\right] N^{\mathcal{B}, i}
$$

The first-order condition for maximization of A 5, with respect to $N^{\mathcal{I}, i}$, is

$$
P^{\mathcal{I}, i}-C^{\mathcal{I}}+P_{1}^{\mathcal{I}, i} N^{\mathcal{I}, i}+P_{2}^{\mathcal{J}, i} N^{\mathcal{J}, i}=0
$$


Note that, by the inverse function theorem, $P_{1}^{\mathcal{I}, i}=\frac{1}{N_{1}^{\mathcal{I}, i}}$, and that by the implicit function theorem, $P_{2}^{\mathcal{I}, i}=-\frac{N_{2}^{\mathcal{I}, i}}{N_{1}^{\mathcal{J}, i}}$. Calculating $N_{1}^{\mathcal{I}, i}$, we find

$$
\begin{aligned}
N_{1}^{\mathcal{I}, i}=- & \underbrace{\int_{-\infty}^{-R^{\mathcal{I},-i}} g^{\mathcal{I}}\left(P^{\mathcal{I}, i}\left(N^{\mathcal{J}, i}\right)-b^{\mathcal{I}} N^{j, i}, B^{\mathcal{I},-i}\right) d B^{\mathcal{I},-i}}_{\text {market expansion }} \\
& -\underbrace{\int_{P^{\mathcal{I}, i}\left(N^{\mathcal{J}, i}\right)-b^{\mathcal{I}} N^{\mathcal{J}, i}}^{\infty} g^{\mathcal{I}}\left(B^{\mathcal{I}, i}, B^{\mathcal{I}, i}+b^{\mathcal{I}} N^{\mathcal{J}, i}-P^{\mathcal{I}, i}\left(N^{\mathcal{J}, i}\right)-R^{\mathcal{I}, i}\right) d B^{\mathcal{I}, i}}_{\text {cannibalization }} .
\end{aligned}
$$

Calculating $N_{2}^{\mathcal{I}, i}$, we find

$$
\begin{aligned}
N_{2}^{\mathcal{I}, i} & =\int_{-\infty}^{-R^{\mathcal{I},-i}} b^{\mathcal{I}} g^{\mathcal{I}}\left(P^{\mathcal{I}, i}\left(N^{\mathcal{J}, i}\right)-b^{\mathcal{I}} N^{j, i}, B^{\mathcal{I},-i}\right) d B^{\mathcal{I},-i} \\
& +\int_{P^{\mathcal{I}, i}(N \mathcal{J}, i)-b^{\mathcal{I}} N^{\mathcal{J}, i}}^{\infty} b^{\mathcal{I}} g^{\mathcal{I}}\left(B^{\mathcal{I}, i}, B^{\mathcal{I}, i}+b^{\mathcal{I}} N^{\mathcal{J}, i}-P^{\mathcal{I}, i}\left(N^{\mathcal{J}, i}\right)-R^{\mathcal{I}, i}\right) d B^{\mathcal{I}, i} \\
& =-b^{\mathcal{I}} N_{1}^{\mathcal{I}, i}
\end{aligned}
$$

Thus, A 6 can be rewritten

$$
P^{\mathcal{I}, i}-C^{\mathcal{I}}+b^{\mathcal{J}} N^{\mathcal{J}, i}=\frac{N^{\mathcal{I}, i}}{-N_{1}^{\mathcal{I}, i}} \equiv \mu_{o}^{\mathcal{I}, i}
$$

where $\mu_{o}^{\mathcal{I}, i}$ is firm $i$ 's own-price market power on side $\mathcal{I}$. Equation (A 7) can be thought of as governing firm $i$ 's best-response to firm $-i$ 's choices of $R^{\mathcal{I},-i}$. Thus the conditions for a symmetric-across-firms (SAF) equilibrium in insulting tariffs, what I call an symmetric insulated equilibrium (SIE), it must be the case that for $I=A, B$ and $i=1,2$

$$
P^{\mathcal{I}}-C^{\mathcal{I}}+b^{\mathcal{J}} \frac{N^{\mathcal{J}}}{2}=\mu_{o}^{\mathcal{I}}
$$

where $P^{\mathcal{I}}$ is the SIE price on side $\mathcal{I}, N^{\mathcal{J}}$ is the total market participation on side $\mathcal{J}$ and $\mu_{o}^{\mathcal{I}}$ is the SAF own-price market power on side $\mathcal{I}$. 


\section{Monopoly}

Suppose firms $i$ and $-i$ can act, via merger or some other means, in such a way so as to maximize joint profits. Then, assuming the platforms choose a SAF strategy, they solve

$$
\max _{N^{\mathcal{I}, m}}\left\{2 \sum_{\mathcal{I}}\left[P^{\mathcal{I}, m}\left(N^{\mathcal{I}, m}, N^{\mathcal{J}, m}\right)-C^{\mathcal{I}}\right] N^{\mathcal{I}, m}\right\}
$$

where $P^{\mathcal{I}, m}\left(N^{\mathcal{I}, m}, N^{\mathcal{J}, m}\right)$ is the inverse, with respect to the first argument, of $N^{\mathcal{I}, m}\left(P^{\mathcal{I}, m}, N^{\mathcal{J}, m}\right)$, the number of side $\mathcal{I}$ agents who join any one of the two symmetric platforms

$$
N^{\mathcal{I}, m}\left(P^{\mathcal{I}, m}\left(N^{\mathcal{J}, m}\right), N^{\mathcal{J}, m}\right)=\int_{P^{\mathcal{I}, m}\left(N^{\mathcal{J}, m}\right)-b^{\mathcal{I}} N^{\mathcal{J}, m}}^{\infty} \int_{-\infty}^{\mathcal{B}^{\mathcal{I}, m}} g^{\mathcal{I}}\left(B^{\mathcal{I}, m}, B^{\mathcal{I},-m}\right) d B^{\mathcal{I},-m} d B^{\mathcal{I}, m}
$$

The first-order condition of the platforms is

$$
P^{\mathcal{I}}-C^{\mathcal{I}}+b^{\mathcal{J}} \frac{N^{\mathcal{J}}}{2}=\frac{N^{\mathcal{I}, m}}{-N_{1}^{\mathcal{I}, m}} \equiv \mu^{\mathcal{I}}
$$

where $\mu^{\mathcal{I}}$ is the joint market power and

$$
N_{1}^{\mathcal{I}, m}=-\int_{-\infty}^{P^{\mathcal{I}, m}-b^{\mathcal{I}} N^{\mathcal{J}, m}} g^{\mathcal{I}}\left(P^{\mathcal{I}, m}-b^{\mathcal{I}} N^{\mathcal{J}, m}, B^{\mathcal{I},-m}\right) d B^{\mathcal{I}-m}
$$

Note that $\mu^{\mathcal{I}}\left(N^{\mathcal{I}}, N^{\mathcal{J}}\right)>\mu_{o}^{\mathcal{I}}\left(N^{\mathcal{I}}, N^{\mathcal{J}}\right)$ for any pair of participation rates $\left(N^{\mathcal{I}}, N^{\mathcal{J}}\right)$ as the denominator of $\mu^{\mathcal{I}}$ does not include the (strictly positive) cannibalization term of $\mu_{o}^{\mathcal{I}}$.

\section{Merger effects}

PROPOSITION 10: Assume 2SC for the Armstrong model applied to the monopoly optimum in this context: $\rho^{\mathcal{I}}>0$ for both $I$ and $\frac{\mu^{\mathcal{A}} \mu^{\mathcal{B}}}{N^{\mathcal{A}} N^{\mathcal{B}}}>\rho^{\mathcal{A}} \rho^{\mathcal{B}}\left(b^{\mathcal{A}}+b^{\mathcal{B}}\right)^{2}$. Then participation and welfare are higher on both sides of the market under duopoly than under monopoly.

\section{PROOF:}

Rewrite condition $A 8$ as

$$
P^{\mathcal{I}}-C^{\mathcal{I}}+b^{\mathcal{J}} N^{\mathcal{J}}=\mu^{\mathcal{I}}-\mu^{\mathcal{I}}\left(1-\frac{M_{o}^{\mathcal{I}}}{M^{\mathcal{I}}}\right)
$$


Let $G^{\mathcal{I}}\left(N^{\mathcal{I}}, N^{\mathcal{J}}\right)=1-\frac{M_{0}^{\mathcal{I}}}{M^{\mathcal{I}}}$ and let the value of $G^{\mathcal{I}}$ at SIE participation rates $\left(N^{\mathcal{A}^{\star}}, N^{\mathcal{B}^{\star}}\right)$ be $G^{\mathcal{I}^{\star}}$. Note that $G^{\mathcal{I}}, G^{\mathcal{I}^{\star}}>0$ as noted above. Inthe monopoly problem costs are given by

$$
C^{\mathcal{I}}-t G^{\mathcal{I}}
$$

for $t \in[0,1]$. Clearly for $t=0$ the solution is the same as the SAF monopoly optimum (which is unique by the assumed second-order conditions). For $t=1$, the allocation must be the same as at the SIE equilibrium. But by my reasoning in Section III.A, participation rates on the two sides of the markets are complements, so a fall in either cost raises both participation rates. Thus an increase in $t$ must raise both participation rates. Therefore participation on both sides of the market is higher under the SIE equilibrium than under the SAF monopoly optimum. Furthermore in the Armstrong model welfare on each side of the market depends (directly) only on participation on that side as there is no Spence distortion, so both groups of users must be better off. Finally, because participation rates are below their optimal level for any amount of market power on both sides, the resulting increase in participation on both sides must be social welfare enhancing. Thus a merger without efficiencies from a SIE equilibrium is harmful to participation, both groups of users' welfare and overall social welfare.

This result contrasts significantly with the RT2003 model, where competition can easily harm one or even both groups of users. Of course the result depends on a particular assumption about tariffs, namely that firms use insulating tariffs. In a previous version of this paper I proved that this result was significantly more robust and holds in a very wide range of reasonable cases with non-insulating tariffs. Those results, omitted for the sake of brevity and clarity, are available on request.

\section{REFERENCES}

Hotelling, Harold. 1929. "Stability in Competition.” Economic Journal, 39(153): 41-57.

Weyl, E. Glen. 2009. “Monopoly, Ramsey and Lindahl in Rochet and Tirole (2003).” Economics Letters, 103(2): 99-100.

Weyl, E. Glen, and Michal Fabinger. 2009. "Pass-Through as an Economic Tool." http://www.fas.harvard.edu/ weyl/research.htm. 